\title{
Embolization of Meningiomas: Comparison of Safety between Calibrated Microspheres and Polyvinyl-Alcohol Particles as Embolic Agents
}

\author{
M. Sluzewski, W.J. van Rooij, P.N. Lohle, G.N. Beute, and J.P. Peluso
}

\begin{abstract}
BACKGROUND AND PURPOSE: During embolization of meningiomas, intratumoral hemorrhagic complications may occur, especially with the use of small particle sizes. We compared the rate of hemorrhagic complications in 55 patients embolized with 400 - $\mu \mathrm{m}$ calibrated microspheres (Embozene) with a historical cohort of 198 patients embolized with smaller PVA particles.
\end{abstract}

MATERIALS AND METHODS: Between September 2009 and February 2012, fifty-five patients with 55 meningiomas were embolized with 400- $\mu \mathrm{m}$ calibrated microspheres. Indications for embolization were preoperative in 47 and before radiosurgery in 2 patients; and in 6 patients, embolization was offered as sole therapy. There were 35 women and 20 men with a mean age of 60.3 years. Mean meningioma diameter was $53 \mathrm{~mm}$ (range, 23-97 mm). Hemorrhagic complications were recorded.

RESULTS: There were no hemorrhagic complications in the 55 embolized patients ( $0 \% ; 95 \% \mathrm{Cl}, 0.0 \%-7.8 \%)$. The difference in complication rates between $400-\mu \mathrm{m}$ calibrated microspheres in this study $(0$ of $55,0 \%)$ and small PVA particles $(45-150 \mu \mathrm{m})$ in the historical cohort (9 of $108,8.3 \%)$ was just short of significance $(P=.066)$. The difference in complication rates between $400-\mu \mathrm{m}$ calibrated microspheres $(0$ of 55 , $0 \%)$ and larger PVA particles $(150-250 \mu \mathrm{m})$ in the historical cohort $(1$ of $93,1.1 \%)$ was not significant $(P=.8)$.

CONCLUSIONS: In this series, embolization of meningiomas by using large $(400-\mu \mathrm{m})$ calibrated microspheres did not result in any hemorrhagic complications.

ABBREVIATIONS: $\mathrm{Cl}=$ confidence interval; PVA = polyvinyl-alcohol

$\mathbf{P}$ reoperative embolization of meningiomas is widely used to facilitate surgical removal and reduce intraoperative blood loss. ${ }^{1-5}$ The potential benefits of preoperative embolization should be balanced against the risk of complications. Recently, several authors have reported a considerable hemorrhagic and ischemic complication rate. ${ }^{6-8}$ In a previous study, we found a $5.1 \%$ hemorrhagic complication rate of embolization of meningiomas in 198 patients, leading to death or dependency in 7, despite emergency surgery. ${ }^{9}$ In that study, the use of small PVA particles $(45-150 \mu \mathrm{m})$ proved to be a significant risk factor for this serious complication. ${ }^{9}$ In view of these findings, we decided to change the embolic material in the embolization of meningiomas, looking for particles as large as conceived feasible. Because large

Received April 26, 2012; accepted after revision June 28

From the Departments of Radiology (M.S., W.J.v.R., P.N.L., J.P.P.) and Neurosurgery (G.N.B.), St. Elisabeth Ziekenhuis, Tilburg, the Netherlands.

Please address correspondence to W.J. van Rooij, MD, Department of Radiology, St. Elisabeth Ziekenhuis, Hilvarenbeekseweg 60, 5022 GC Tilburg, the Netherlands; e-mail: radiol@eztilburg.nl

- Indicates open access to non-subscribers at www.ajnr.org

http://dx.doi.org/10.3174/ajnr.A3311
PVA particles have the tendency to block the microcatheter, we opted for the use of calibrated microspheres. Large-sized calibrated microspheres are easily compressible and smooth, and these physical properties prevent blockage of the microcatheter. ${ }^{10}$

In the present study, we assessed the frequency of hemorrhagic complications in 55 embolized meningiomas in 55 patients by using 400- $\mu \mathrm{m}$ calibrated microspheres (Embozene microspheres; CeloNova BioSciences, Newnan, Georgia) and compared this complication rate with that of 198 patients previously embolized with PVA. ${ }^{9}$

\section{MATERIALS AND METHODS \\ General}

Between September 2009 and February 2012, fifty-five patients with 55 meningiomas were embolized in our institution. Referral for preoperative embolization of patients with meningiomas was dependent on the personal preference of the neurosurgeon. Indications for embolization were preoperative in 47 and before radiosurgery in 2 patients. In the remaining 6 patients, embolization was initially offered as a sole therapy. The time interval between embolization and surgery was 1 day in all surgical patients.

We assessed the following patient and imaging characteristics: 
age, sex, indication for embolization, and meningioma size. Meningioma location was categorized as convexity, falx, sphenoid ridge, posterior fossa, and tentorial. Peritumoral edema was dichotomized into minor and major (with midline shift). Arterial supply to the meningioma was classified as predominantly external carotid artery supply, equal external and internal carotid artery supply, and predominantly internal carotid artery supply. Vascularization of the meningioma was first assessed by angiography of the internal and external carotid arteries and/or the vertebral artery. Embolization was performed via the middle meningeal or occipital artery only. The ophthalmic artery, the meningeohypophyseal trunk, or pial feeders were not used as access for embolization because of possible ischemic complications in these vascular territories. Hemorrhagic complications, defined as any extravasation of contrast material visible during embolization and confirmed on CT scanning, were recorded. Also ischemic complications and blockage of the microcatheter were noted.

Data of our previous study of 198 patients with 201 embolized meningiomas were used for comparison. In that study, embolization was performed with either 45- to $150-\mu \mathrm{m}$ PVA particles or 150- to $250-\mu \mathrm{m}$ PVA particles. The smaller PVA particles were used in 108 meningiomas (54\%), and the larger, in 93 meningiomas $(46 \%){ }^{9}$

Patient and imaging characteristics of the previous study were compared with those of the present study. We recalculated the hemorrhagic complication rates for both PVA particle sizes in the previous study and compared these with the hemorrhagic complication rates of 55 meningiomas embolized with $400-\mu \mathrm{m}$ calibrated microspheres in the present study.

\section{Embolization Technique}

Angiography and embolization were performed with the patient awake. Patients were premedicated with steroids. No intravenous heparin was administered. Embolization was performed via the middle meningeal or occipital artery only. The ophthalmic artery, the meningeohypophyseal trunk, or pial feeders were not used as access for embolization to avoid ischemic damage to cranial nerves or brain. Embolization was performed through a standard microcatheter (Turbo Tracker 18 or Excel 14; Boston Scientific, Fremont, California) by using $400-\mu \mathrm{m}$ calibrated microspheres (Embozene microspheres). These microspheres consist of a hydrogel core of polymethylmethacrylate and a flexible shell of polyphosphazene, which is a synthesized inorganic biostable and biocompatible polymer. The microspheres suspended in contrast material are spherical, flexible, and easily compressible. Embozene microspheres are precisely calibrated by sieving with a high uniformity of spheres.

These physical properties of the microspheres prevent clogging (as do PVA particles), even in small-lumen microcatheters. This feature is important because with the use of larger microcatheters, distal navigation may be hindered. The microspheres are color-coded according to size and are available in sizes ranging from 40 to $1300 \mu \mathrm{m}$. The $400-\mu \mathrm{m}$ Embozene microspheres are blue.

Under fluoroscopic control, the mixture of contrast material and microspheres was slowly injected until stagnation of the contrast agent in the feeding artery was accomplished.

\section{Data Analysis}

Data are summarized as descriptive statistics. In addition, a $\chi^{2}$ test was used for comparison of proportions, and an unpaired $t$ test, for comparison of means.

\section{RESULTS}

Of the 55 patients, 35 were women and 20 were men, with a mean age of 60.3 years (median, 62 years; range, $31-87$ years). Mean meningioma axial diameter was $53 \mathrm{~mm}$ (median, $51 \mathrm{~mm}$; range, 23-97 mm). Meningioma location was convexity in 36, falx in 9, sphenoid ridge in 4 , posterior fossa in 3 , and tentorial in 3 . Minor or no peritumoral edema was seen in 27 meningiomas (50\%); major edema with a shift of midline structures was present in 28 meningiomas (50\%). Arterial supply was predominantly external carotid artery in 36 meningiomas (65\%) and equally external and internal carotid arteries in 19 meningiomas (35\%). Patient and imaging characteristics of the 55 patients in the present study were not different from those of the 198 patients in the previous study.

In 55 patients with embolized meningiomas, no hemorrhagic complications occurred (0\%; 95\% CI, $0.0 \%-7.8 \%)$. Also ischemic complications did not occur. There were no instances of blockage of the microcatheter. Recalculation of hemorrhagic complication risks in our previous study was as follows: There were 9 hemorrhagic complications in the 108 meningiomas embolized with 45to $150-\mu \mathrm{m}$ PVA particles $(8.3 \%$; $95 \% \mathrm{CI}, 4.3 \%-15.2 \%)$. There was 1 hemorrhagic complication in 93 meningiomas embolized with 150 - to $250-\mu \mathrm{m}$ PVA particles $(1.1 \%$; $95 \%$ CI, $<0.0001 \%-6.4 \%$ )

The difference in complication rates between the use of $400-\mu \mathrm{m}$ calibrated microspheres in this study ( 0 of $55,0 \%)$ and the use of small PVA particles $(45-150 \mu \mathrm{m})$ in the previous study ( 9 of 108, 8.3\%) was just short of significance $(P=.066$ ).

The difference in complication rates between the use of $400-\mu \mathrm{m}$ calibrated microspheres in this study (0 of 55, $0 \%$ ) and the use of larger PVA particles $(150-250 \mu \mathrm{m})$ in the previous study ( 1 of $93,1.1 \%)$ was not significant $(P=.8)$.

\section{DISCUSSION}

In this study, we found that the use of $400-\mu \mathrm{m}$ calibrated microspheres in the embolization of meningiomas was safe; no hemorrhagic complications occurred. These results compare favorably with the hemorrhagic complication rate of $8.3 \%$ in our previous study with use of small PVA particles of $45-150 \mu \mathrm{m} .{ }^{9}$ There was no difference in complication rates between the use of $400-\mu \mathrm{m}$ calibrated microspheres and the use of larger PVA particles of $150-250 \mu \mathrm{m}$.

The prevention of hemorrhagic complications in the embolization of meningiomas is important because intratumoral hemorrhage may have serious consequences. Extravasation of blood into the tumor causes acute edema and swelling of the meningioma with sudden aggravation of mass effect. Many patients who are referred for preoperative meningioma embolization have large tumors with little skull volume reserve capacity, and even a small increase in tumoral volume may lead to tentorial hernia- 
tion. The pathophysiology of hemorrhage in meningiomas that are embolized is not fully understood. It has been postulated that necrosis as a result of deep penetration of the particles causes the tissue to be more vulnerable to bleeding. ${ }^{6-8,11,12}$ Possibly, penetration of particles into the draining veins of the tumor may block the outflow, increasing the risk of hemorrhage.

The possibility that small particles may carry a potentially higher risk for intratumoral hemorrhage in the embolization of meningiomas was addressed by several authors, some even referring to these small particles as being "aggressive" or "dangerous." ${ }^{\prime, 13}$ On the other hand, small particles may be more effective due to a better devascularization of the tumor compared with larger particles. ${ }^{9,12}$ Despite these contradictory considerations, we believe that embolization of meningiomas, especially when performed preoperatively, should carry the lowest possible risk. We, therefore, have completely refrained from the use of small 45to $150-\mu \mathrm{m}$ PVA particles. Our reason for choosing and evaluating 400- $\mu \mathrm{m}$ calibrated microspheres was 2 -fold. First, the calibrated microspheres of $400 \mu \mathrm{m}$ are much larger than the 45 - to 150 - and 150- to 250- $\mu \mathrm{m}$ PVA particles we used previously. Second, these microspheres suspended in contrast material are spheric, smooth, flexible, easily compressible, and precisely calibrated; and these physical properties enhance the ease of use without the occurrence of catheter blockage. Although we did not specifically address the topic of effectiveness of devascularization in terms of quantification of blood loss or operation time, neither we nor our neurosurgeons noticed any difference between PVA and microspheres.

Our study has several limitations. Due to the retrospective nature of the earlier series and the differences in properties of the calibrated microspheres, the 2 groups may not be completely comparable. On the other hand, referral pattern, indication, catheterization technique, and imaging methods did not change throughout the entire study period, only the embolic agent used. In this respect, we think that comparison of the groups is valid.

Eight of 55 patients had no MR imaging or CT directly after embolization of the meningioma. These 8 patients were clinically unchanged after embolization without suspicion of a hemorrhagic complication. However, we cannot be sure that small asymptomatic intratumoral hemorrhage did not occur.

\section{CONCLUSIONS}

In the embolization of meningiomas, the use of large particles such as $400-\mu \mathrm{m}$ calibrated microspheres is safer than the use of small PVA particles. No hemorrhagic complications occurred with microspheres as embolic agents.

Disclosures: Paul N. Lohle-UNRELATED: Grants/Grants Pending: Stryker;* Travel/ Accommodations/Meeting Expenses Unrelated to Activities Listed: Stryker, CeloNova, Merit Biosphere Medical, Cook Medical, Comments: Travel and accommodation expenses are sometimes reimbursements for giving presentations or workshops (on vertebroplasty, fibroid, and adenomyosis embolization) during international interventional congresses. *Money paid to the institution.

\section{REFERENCES}

1. Richter HP, Schachenmayr W. Preoperative embolization of intracranial meningiomas. Neurosurgery 1983;13:261-68

2. Manelfe C, Lasjaunias P, Ruscalleda J. Preoperative embolization of intracranial meningiomas. AJNR Am J Neuroradiol 1986;7:963-72

3. Macpherson $P$. The value of pre-operative embolisation of meningioma estimated subjectively and objectively. Neuroradiology 1991;33:334-37

4. Dean B, Flom RA, Wallace RC, et al. Efficacy of endovascular treatment of meningiomas: evaluation with matched samples. AJNR Am J Neuroradiol 1994;15:1675-80

5. Gruber A, Killer M, Mazal P, et al. Pre-operative embolization of intracranial meningiomas: a 17-year single center experience. Minim Invasive Neurosurg 2000;43:18-29

6. Bendszus M, Monoranu CM, Schutz A, et al. Neurologic complications after particle embolization of intracranial meningiomas. AJNR Am J Neuroradiol 2005;26:1413-19

7. Yu SC, Boet R, Wong GK, et al. Postembolization hemorrhage of a large and necrotic meningioma. AJNR Am J Neuroradiol 2004;25:506-08

8. Kallmes DF, Evans AJ, Kaptain GJ, et al. Hemorrhagic complications in embolization of a meningioma: case report and review of the literature. Neuroradiology 1997;39:877-80

9. Carli DF, Sluzewski M, Beute GN, et al. Complications of particle embolization of meningiomas: frequency, risk factors, and outcome. AJNR Am J Neuroradiol 2010;31:152-54

10. Verret V, Ghegediban SH, Wassef M, et al. The arterial distribution of Embozene and Embosphere microspheres in sheep kidney and uterus embolization models. J Vasc Interv Radiol 2011;22:220-28

11. Wakhloo AK, Juengling FD, Van Velthoven V, et al. Extended preoperative polyvinyl alcohol microembolization of intracranial meningiomas: assessment of two embolization techniques. AJNR Am J Neuroradiol 1993;14:571-82

12. Bendszus $M$, Rao $G$, Burger $R$, et al. Is there a benefit of preoperative meningioma embolization? Neurosurgery 2000;47:1306-11, discussion 1311-12

13. Latchaw RE. Preoperative intracranial meningioma embolization: technical considerations affecting the risk-to-benefit ratio. AJNR Am J Neuroradiol 1993;14:583-86 